\title{
Non-Real-Time Network Traffic in Software-Defined Networking: A Link Bandwidth Prediction-Based Algorithm
}

\author{
Longfei Dai ${ }^{1}$, Wenguo Yang ${ }^{1}$, Suixiang Gao ${ }^{1}$, Yinben $\mathrm{Xia}^{2}$, Mingming $\mathrm{Zhu}^{2} \&$ Zhigang $\mathrm{Ji}^{2}$ \\ ${ }^{1}$ School of Mathematical Sciences, University of Chinese Academy of Sciences, Beijing, China \\ ${ }^{2}$ Huawei Technologies Co.LTD., Beijing, China \\ Correspondence: Longfei Dai, School of Mathematical Sciences, University of Chinese Academy of Sciences, \\ 19A Yuquan Road, Beijing, China. Tel: 86-188-1040-0940. E-mail: dailongfei11@mails.ucas.ac.cn
}

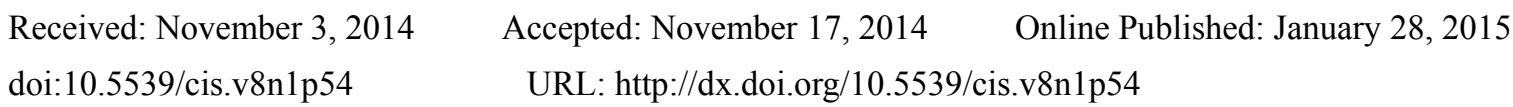

The research is financed by 973 project (2011CB706901) and Huawei Technology Co. Ltd.

\begin{abstract}
Network traffic control is the process of managing, prioritizing, controlling or reducing the network traffic by the network scheduler. High utilization of link bandwidth is very significant for network control and maintenance in Software-Defined Networking (SDN). When we get the accurate link bandwidth predictions for $T$ time periods of the future in a specific network topology, the residual link bandwidth could be determined by the link bandwidth capacity and corresponding prediction values. Given the non-real-time request pairs, this process can be transformed into a multi-commodity flow model. But the traditional multi-commodity model has not introduced the time dimension. In this paper, the model associated with the time dimension is to complete the transmission of the non-real-time network traffic. However, in consideration of the large scale of the problem, a heuristic algorithm on the basis of greedy strategy is proposed to schedule the non-real-time network traffic properly. The experiments show that the heuristic algorithm is superior to global optimization in computing speed and the single path resulting from heuristic algorithm occupies fewer links in the network topology for the non-real-time network traffic.
\end{abstract}

Keywords: SDN, network control, network traffic prediction, multi-commodity model

\section{Introduction}

As the number of Internet users and all kinds of network services grows rapidly, many new problems are led by the drastic data flows of the internet (K. Hao, Z. Jin \& P. Hao, 2012). The main problem is the network congestion which seriously affects the packet transmission rate and the network operating quality (Bing, Lu \& Long, 2008). Although network congestion might be have many causes such as limited storage space for node, bad construction of the network topology or the trouble of the routing method etc., the core reason is that bandwidth capacity and the device processing ability provided by network could not satisfy the requirements while the growth of application traffic is approximately unlimited. In the network, "queue management" is the main approach to solve the congestion control problem and Mohammad M. H. Y (2010) proposed a fuzzy active queue management for internet congestion control. Random Early Detection (RED) (Floyd \& Jacobson, 1993) is a very popular and important algorithm of the active queue management and all variants of the algorithms based RED are proposed such as Stabilized RED (Ott, Lakshman \& Wong, 1999), Dynamics of Random Early Detection (Lin \& Morris, 1997) and Adaptive RED (Floyd, Gummadi \& Shenker, 2001). But active queue management algorithm exist many shortcomings especially in the detection phase (Papadimitriou, Welzl, Scharf \& Briscoe, 2011). Another approach for network control is TCP congestion control. Research by Dassouki, Debar, Safa and Hijazi (2013) proposed a mechanism which is capable of detecting congestions by monitoring passively an aggregation link. Moreover, the increases of the network scale enhance the complexity so that the design for more effective network congestion control strategy is confronted with great challenge.

In order to better fulfill the development of network requirements, a new configuration model of network called Software-Defined Networking (SDN) is proposed in recent years. SDN is an emerging architecture purporting to be dynamic, manageable, cost-effective, and adaptable, seeking to be suitable for the high-bandwidth, dynamic 
nature of today's applications. SDN architectures decouple network control and forwarding functions, enabling network control to become directly programmable and the underlying infrastructure to be abstracted from applications and network services, which can treat the network as logical or virtual entity (Open Networking Foundation [ONF], 2013). SDN introduces new possibilities for changing business needs which are more convenient and flexible to control the network traffic (McKeown et al., 2008).

Whereas the goal of the SDN is global optimization of the network, it is obvious that network traffic control is still an important issue in the network management with the sustained expanding of network scale and emergence of lots of real-time service request. In addition, with the rapid development of datacenters, large amounts of non-real-time network traffic should be transmitted or communicated between the datacenters, such as data backup traffic and content synchronization traffic (Jia \& Wang, 2013). The optimization of the network link transmission efficiency could meet the traffic demands and attaining a low congestion as well as high-efficient utilization of network resources (Huerta, Hesselbach \& Fabregat, 2006).

Recognizing the distinctions of the real-time and non-real-time network traffic (Wijnants \& Lamotte, 2008) in the process of transmission, different types of network traffic utilize different transmission modes inspired by the particular characteristics of the traffic. In the network, each link has a bandwidth capacity and parts of the bandwidth are occupied by the real-time network traffic. In general, real-time users' traffic fluctuates over time. Therefore, based on the change trend of real-time traffic predicted by network traffic prediction algorithm, we could control non-real-time network traffic to transmit at the time when there is a relatively small amount of real-time traffic in the network. Based on the above analysis, we can take full advantage of the idle bandwidth resources to complete the transmission of non-real-time network traffic while the real-time user's action will not be affected. Because of the not too strict time limitation, the links are not occupied too much in transmitting nor-real-time network traffic. To solve the issue, a heuristic algorithm on the basis of greedy strategy is proposed to schedule the non-real-time network traffic properly.

The rest of the paper is organized as follows. Section 2 analyzes the predictability of the real-time network traffic. In section 3, we depict the mathematical model of the problem as well as the corresponding proposed algorithm. And in section 4 our experimental results are presented. Finally, we draw a conclusion in section 5.

\section{Real-Time Network Predictions}

Real-time network prediction is an important issue in the network management. As the importance and the extensive potential applications of network traffic prediction in SDN, a lot of network traffic prediction models or algorithms have been investigated by researchers.

In 1993, Ethernet LAN traffic was demonstrated to have statistically self-similar (Leland, Taqqu, Willinger \& Wilson, 1993). Network traffic is essentially a stochastic time series. Many prediction models have been created for decades with the development of the time series analysis. The time series model proposed by Box-Jenkins (Box, Jenkins \& Reinsel, 2013) supplied a solution for the linear stationary process by Auto-Regressive (AR), Moving Average (MA) and the combination of Auto-Regressive and Moving Average (ARMA). Recently, as the fast development in Artificial Intelligence, many intelligent algorithms, such as Wavelet Analysis, Artificial Neural Networks (Kantz \& Schreiber, 2004), and Support Vector Machine (SVM) are widely used in forecasting (Cristianini \& Shawe-Taylor, 2000). Besides, many hybrid algorithms integrate the advantages of various algorithms to attain better prediction effect.

As the real-time network traffic consists of the group behavior of the users, real-time network traffic will appear some regularity. Thus we can design the proper model to predict the real-time network traffic changes in the future. In this paper, we assume that we could accurately predict the future $T$ time interval network traffic of every link and distribute the non-real-time network traffic to the residual network. This kind of strategies that transmit the non-real-time network traffic can take full advantage of the idle link bandwidth, and decrease the cost of the network transmission.

\section{Model Descriptions and Algorithm}

In this section, firstly we depict the problem with the directed acyclic graph and give the mathematical model of the problem. Then according to the characteristic of the problem a routing selection algorithm on the basis of link capacity of the bandwidth is proposed in part 3.2.

\subsection{Mathematical Model}

In the process of transmitting the real-time service, we assume the SDN topology of the network is a directed acyclic graph $G=(V, E, c, w)$, where $V$ denotes the set of vertices, $E$ denotes the set of edges or links, $c(e)$ denotes the link bandwidth of the edge $e$ and $w(e)$ represents the cost of every link for transmission. Because the network 
traffic of each link is obtained by periodic sampling, a maximum number $T$ is given as the non-real-time network traffic will be transmitted in $T$ periods of the future. The purpose of non-real-time traffic control is to optimize the transmission path with the time and link bandwidth limit, where every time period represented by $\Delta t$ is identified as unite of time, i.e., $\Delta t=1$.

Suppose the $k$ th request pair is $r_{k}=\left(s_{k}, t_{k}, d_{k}\right)$ and denotes its flow function by $f_{k p}$ at the $p$ th time period, where $p=1$, $2 \ldots T$ and $k=1,2, \ldots n$. We denote the real-time network traffic by $X_{p}(e)$ for $e \in E$ in time period $p$. Then the link bandwidth which could be used for non-real-time network traffic transmission is:

$$
c_{p}(e)=c(e)-X_{p}(e), e \in E, p=1,2 \ldots T .
$$

When given the specific network and the number of time period, a mathematical model based on multi-commodity problem with time window constraint is described as follows:

$$
\min \sum_{p=1}^{T} \sum_{e \in E} w(e)\left(\sum_{k=1}^{n} f_{k p}(e)\right)
$$

s.t.

$$
\begin{aligned}
& \sum_{k=1}^{n} f_{k p}(e) \leq c_{p}(e), \text { for } e \in E \text { and } 1 \leq p \leq T \\
& \sum_{p=1}^{T} \sum_{\left(s_{k}, y\right) \in E} f_{k p}\left(s_{k}, y\right)=\sum_{p=1}^{T} \sum_{\left(x, t_{k}\right) \in E} f_{k p}\left(x, t_{k}\right)=d_{k}, \quad 1 \leq k \leq n \\
& \sum_{p=1}^{T} \sum_{\left(x, s_{k}\right) \in E} f_{k p}\left(x, s_{k}\right)=\sum_{p=1}^{T} \sum_{\left(t_{k}, y\right) \in E} f_{k p}\left(t_{k}, y\right)=0, \quad 1 \leq k \leq n \\
& \sum_{(u, v) \in E} f_{k p}(u, v)=\sum_{(v, u) \in E} f_{k p}(v, u), \text { for } u \in V /\left\{s_{k}, t_{k}\right\}, 1 \leq p \leq T, 1 \leq k \leq n \\
& f_{k p}(e) \geq 0, \quad \text { for } e \in E, 1 \leq k \leq n, 1 \leq p \leq T
\end{aligned}
$$

The objective function expresses the total cost of the traffic transmission. Constraint (1) implies that the total flow of a link $e$ should not exceed the capacity $c_{p}(e)$. Constraints $(2) \sim(4)$ indicate that for every network node the traffic must satisfy the flow conservation. Constraint (5) assures the non-negativity of the variables.

\subsection{Algorithm Description}

Problem $(\mathrm{P})$ is a multi-commodity model essentially with time dimension which augments the complexity of the computation to solve a linear programming, although many accurate algorithms could get optimal solution. In order to meet the transmission requirements of non-real-time network traffic rapidly and make full use of the link bandwidth, we design a heuristic routing selection algorithm on the basis of link capacity to get a non-real-time network traffic scheme according to the characteristics of the problem. In the heuristic algorithm, we introduce the concept of priority associated with the demand $d_{k}$ of require $r_{k}(k=1, \ldots, n)$. Given a priority function denoted by $\operatorname{prio}(\cdot)$, for all $d_{i}>d_{j}, \operatorname{prio}\left(r_{i}\right)>\operatorname{prio}\left(r_{j}\right)$ if and only if require $r_{i}$ is first transmitted in the algorithm (breaking ties arbitrarily).

For any link $e \in E$ in $G$, given that $X_{p}(e)$ denotes the bandwidth already used in the link $e$ and the residual bandwidth of link $e$ denotes by $c_{p}(e)=c(e)-X_{p}(e)$ in the $p$ th time interval. Therefore, the residual network is defined $G_{p}=\left(V, E, c_{p}, w\right)$ but with the bandwidth capacity $c_{p}(e)$. The major steps of the proposed heuristic algorithm are illustrated below:

Step 1. Suppose the historical network traffic time series of each link $e \in E$ have been collected and then calculate the predicted value $X_{p}(e)$ of time period from $p=1$ to $T$.

Step 2. For any $e \in E$ in $G$, according to the predicted value $X_{p}(e)$ of each link in the forecasting period $p$, it is convenient to obtain the residual bandwidth of each link. Intuitively the residual bandwidth of each link is depicted as:

$$
c_{p}(e)=c(e)-X_{p}(e), p=1, \ldots, T .
$$

Step 3. Now the residual network $G_{p}=\left(V, E, c_{p}, w\right)(p=1, \ldots, T)$ covers the whole process of the network transmission in the $T$ time periods. And in every time period p, employing the path selection algorithm the non-real-time network traffic will be distributed on each link of the topology $G_{p}$.

In the $T$ time periods, Without loss of generality, we assume that if $i<j$, prio $\left(r_{i}\right)>$ prio $\left(r_{j}\right)$. Under the definition of priority the specific network traffic loading process is as follows: 
Step 3.0 Initialization of $i=0$. Define $\mathrm{c}_{i p}(e)(i=1,2, \ldots, n)$ as the residual bandwidth which is about to transmit the require $r_{i}$ at the $p$ th $(p=1, \ldots, T)$ time period for $e \in E$.

Step 3.1 Set $i=i+1$ and $p=0$.

(1) Set $p=p+1$, the residual bandwidth is $\mathrm{c}_{i p}(e)$ right now. Then calculate the routing path of request $r_{i}$ according to the procedure (*), and denote $d_{i p}$ as the maximal traffic in the routing path;

(2) Set $d_{i}=d_{i}-d_{i p}$,

If $d_{i} \leq 0$ and $p \leq T$, then succeed to transmit request $r_{i}$, go to step 3.2;

If $d_{i}>0$ and $p<T$, go to step (1);

If $d_{i}>0$ and $p \geq T$, then cannot complete the transmission of request $r_{i}$ within the $T$ time periods and abandon the request $r_{i}$ from the transmission queue. Then set $d_{i p}=0$ and go to step 3.2.

Step 3.2 If $i=n$, then stop. Otherwise, for all $p=1, \ldots, T$ and $e \in E$ :

$$
c_{(i+1) p}=\mathrm{c}_{i p}(e)-d_{i p},
$$

Go to step 3.1.

\section{Procedure (*) Routing Selecting Algorithm}

Input: The directed graph $G$ with capacity constraint cap;

The source node $s$ and the terminal node $t$.

Output: The flow and path from $s$ to $t$.

Step 1 Let label $(s)=\infty$, label $(v)=0(v \neq s)$, maxcap $=0$;

Let $\mathrm{Q}$ is a First-in First-Out queue of candidate vertices, push s to Q;

Step 2 while $\mathrm{Q}$ is not empty

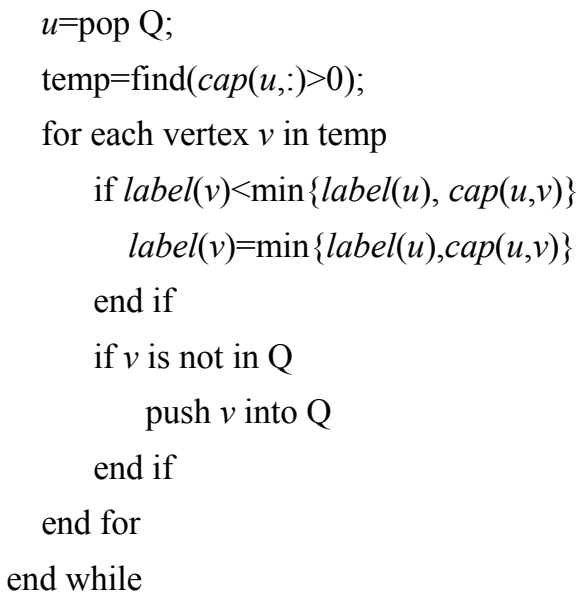

Step 3 traverse from the $t$ to the $s$ to get the path;

Maxcap $=l a b e l(t)$.

\section{Experiments and Results}

\subsection{Experiments Simulation}

In this part, the Internet2 network topology is given to demonstrate the validity and high-efficiency of the proposed algorithm by the simulation results. The Internet 2 backbone network has nine main nodes and also denotes it by $G=(V, E, c, w)$ where the $w$ is determined by the transmission distance. The values of $w$ are illustrated in table 1 . In the experiments, the number of time period $T$ is set to 8 and the request pair number is two; i.e. $r_{1}=\left(s_{1}, t_{1}, d_{1}\right)=(1,5,2200)$ and $r_{2}=\left(s_{2}, t_{2}, d_{2}\right)=(3,7,2000)$. In order to simplify the description of the process, the time interval $\Delta t$ is consider as the unit of the time as before, i.e., $\Delta t=1$ in this paper. 
Table 1. The values of $w$ of each Link

\begin{tabular}{llllllllll}
\hline$w$ & v1 & v2 & v3 & v4 & v5 & v6 & v7 & v8 & v9 \\
\hline v1 & 0 & 0 & 0 & 0 & 0 & 0 & 1.342 & 0.913 & 0 \\
v2 & 0 & 0 & 1 & 0.905 & 1.045 & 0 & 0 & 0 & 0.69 \\
v3 & 0 & 1 & 0 & 0.278 & 0 & 0 & 0 & 0 & 0 \\
v4 & 0 & 0.905 & 0.278 & 0 & 0.7 & 0 & 0 & 0 & 0 \\
v5 & 0 & 1.045 & 0 & 0.7 & 0 & 1.385 & 0 & 0 & 0 \\
v6 & 0 & 0 & 0 & 0 & 1.385 & 0 & 1.705 & 0 & 0.818 \\
v7 & 1.342 & 0 & 0 & 0 & 0 & 1.705 & 0 & 1.303 & 0 \\
v8 & 0.913 & 0 & 0 & 0 & 0 & 0 & 1.303 & 0 & 1.33 \\
v9 & 0 & 0.69 & 0 & 0 & 0 & 0.818 & 0 & 1.33 & 0 \\
\hline
\end{tabular}

Although network traffic prediction of the link is essential in transmitting the non-real-time network traffic, prediction is a relatively independent process so that we only need to know the residual bandwidth $c_{p}(e)$ of each link $e$ at every time period in the particular network topology. Because the SDN has the global control ability in the network, the size of the $c_{p}(e)$ will also be influenced by the SDN controller. To simulate the process of SDN controller, the size of $c_{p}(e)$ is generated randomly between 100 to 600 which is illustrated in the table 2. Residual bandwidth $c_{p}(e)$ of each link will be used to transmit the non-real-time network traffic in the given time period $p$ $(p=1, \ldots, 8)$.

Table 2. The residual bandwidth of each link in each period

\begin{tabular}{|c|c|c|c|c|c|c|c|c|}
\hline & $\mathrm{T} 1$ & $\mathrm{~T} 2$ & T3 & $\mathrm{T} 4$ & T5 & T6 & $\mathrm{T} 7$ & $\mathrm{~T} 8$ \\
\hline$(\mathrm{v} 1, \mathrm{v} 7)$ & 465 & 469 & 443 & 591 & 594 & 342 & 311 & 358 \\
\hline$(\mathrm{v} 1, \mathrm{v} 8)$ & 111 & 441 & 312 & 522 & 150 & 441 & 171 & 160 \\
\hline$(\mathrm{v} 2, \mathrm{v} 3)$ & 450 & 418 & 460 & 439 & 410 & 249 & 145 & 365 \\
\hline$(\mathrm{v} 2, \mathrm{v} 4)$ & 116 & 212 & 282 & 448 & 592 & 366 & 447 & 471 \\
\hline$(\mathrm{v} 2, \mathrm{v} 5)$ & 527 & 557 & 146 & 363 & 250 & 401 & 343 & 567 \\
\hline$(\mathrm{v} 2, \mathrm{v} 9)$ & 449 & 218 & 477 & 161 & 334 & 470 & 192 & 426 \\
\hline$(\mathrm{v} 3, \mathrm{v} 2)$ & 468 & 176 & 159 & 474 & 420 & 146 & 420 & 177 \\
\hline$(\mathrm{v} 3, \mathrm{v} 4)$ & 380 & 433 & 481 & 391 & 529 & 154 & 479 & 312 \\
\hline$(\mathrm{v} 4, \mathrm{v} 2)$ & 234 & 240 & 569 & 512 & 308 & 253 & 342 & 290 \\
\hline$(\mathrm{v} 4, \mathrm{v} 3)$ & 369 & 220 & 596 & 194 & 126 & 352 & 152 & 398 \\
\hline$(\mathrm{v} 4, \mathrm{v} 5)$ & 288 & 331 & 365 & 500 & 266 & 464 & 298 & 220 \\
\hline$(\mathrm{v} 5, \mathrm{v} 2)$ & 311 & 320 & 422 & 494 & 202 & 328 & 175 & 180 \\
\hline$(\mathrm{v} 5, \mathrm{v} 4)$ & 434 & 272 & 485 & 539 & 356 & 269 & 427 & 162 \\
\hline$(\mathrm{v} 5, \mathrm{v} 6)$ & 245 & 336 & 231 & 141 & 188 & 403 & 448 & 511 \\
\hline$(\mathrm{v} 6, \mathrm{v} 5)$ & 314 & 330 & 342 & 349 & 424 & 490 & 118 & 479 \\
\hline$(\mathrm{v} 6, \mathrm{v} 7)$ & 539 & 194 & 452 & 108 & 307 & 136 & 415 & 439 \\
\hline$(\mathrm{v} 6, \mathrm{v} 9)$ & 259 & 329 & 574 & 121 & 468 & 533 & 453 & 497 \\
\hline$(\mathrm{v} 7, \mathrm{v} 1)$ & 344 & 405 & 391 & 542 & 526 & 126 & 485 & 442 \\
\hline$(\mathrm{v} 7, \mathrm{v} 6)$ & 232 & 270 & 477 & 430 & 265 & 152 & 165 & 181 \\
\hline$(\mathrm{v} 7, \mathrm{v} 8)$ & 147 & 417 & 295 & 281 & 187 & 264 & 225 & 190 \\
\hline$(\mathrm{v} 8, \mathrm{v} 1)$ & 269 & 489 & 370 & 394 & 536 & 320 & 202 & 301 \\
\hline$(\mathrm{v} 8, \mathrm{v} 7)$ & 230 & 145 & 109 & 531 & 481 & 499 & 167 & 504 \\
\hline$(\mathrm{v} 8, \mathrm{v} 9)$ & 427 & 485 & 435 & 240 & 192 & 283 & 256 & 320 \\
\hline$(\mathrm{v} 9, \mathrm{v} 2)$ & 591 & 359 & 423 & 155 & 171 & 248 & 218 & 442 \\
\hline$(\mathrm{v} 9, \mathrm{v} 6)$ & 591 & 195 & 321 & 545 & 159 & 374 & 103 & 547 \\
\hline$(\mathrm{v} 9, \mathrm{v} 8)$ & 335 & 204 & 298 & 344 & 552 & 474 & 451 & 110 \\
\hline
\end{tabular}

Note. (v1, v7) represent links (similarly hereinafter) and T1 T8 denote time intervals.

The optimal solution is acquired by using the GNU Linear Programming Kit (GLPK) software package. In the following figure, the routings and flow of the optimal solution (Opt) and the Heuristic Algorithm (HA) are given from the figure 1 to figure 8 of the transmission process. 


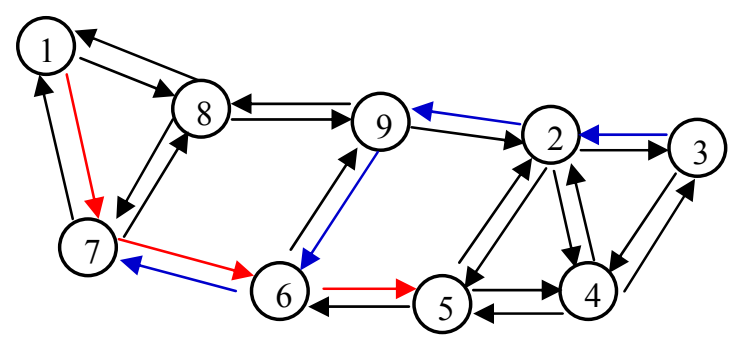

Figure 1. the Transmission of Period 1

Note. HA: $1 \rightarrow 7 \rightarrow 6 \rightarrow 5$ (232), $3 \rightarrow 2 \rightarrow 9 \rightarrow 6 \rightarrow 7$ (449); Opt: $1 \rightarrow 8 \rightarrow 9 \rightarrow 2 \rightarrow 5$ (111), $1 \rightarrow 7 \rightarrow 6 \rightarrow 5$ (88) $3 \rightarrow 2 \rightarrow 9 \rightarrow 6 \rightarrow 7(294), 3 \rightarrow 4 \rightarrow 5 \rightarrow 6 \rightarrow 7(245)$

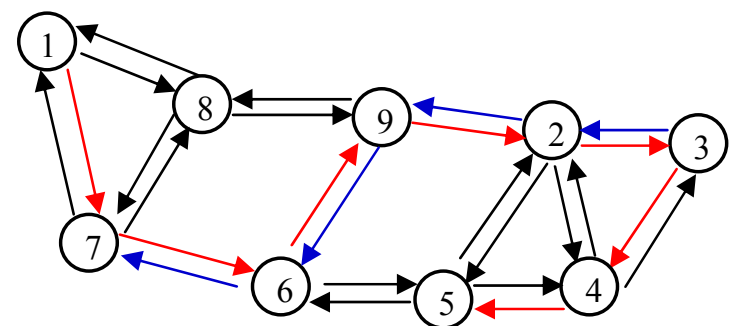

Figure 3. the Transmission of Period 3

Note. HA: $1 \rightarrow 7 \rightarrow 6 \rightarrow 9 \rightarrow 2 \rightarrow 3 \rightarrow 4 \rightarrow 5$ (365), $3 \rightarrow 2$ $\rightarrow 9 \rightarrow 6 \rightarrow 7$ (159); Opt: $1 \rightarrow 8 \rightarrow 9 \rightarrow 2 \rightarrow 5$ (146), $1 \rightarrow$ $7 \rightarrow 6 \rightarrow 5(342), 3 \rightarrow 4 \rightarrow 5 \rightarrow 6 \rightarrow 7(231)$

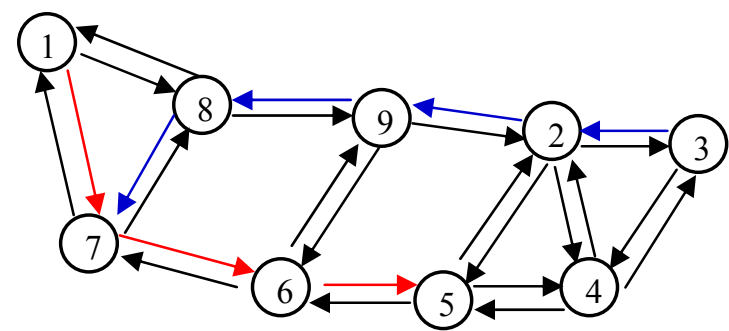

Figure 5. the Transmission of Period 5

Note. HA: $1 \rightarrow 7 \rightarrow 6 \rightarrow 5$ (265), $3 \rightarrow 2 \rightarrow 9 \rightarrow 8 \rightarrow 7$ (334); Opt: $1 \rightarrow 8 \rightarrow 9 \rightarrow 2 \rightarrow 5$ (150), $3 \rightarrow 2 \rightarrow 9 \rightarrow 6 \rightarrow$ $7(86), 3 \rightarrow 4 \rightarrow 5 \rightarrow 6 \rightarrow 7(188)$

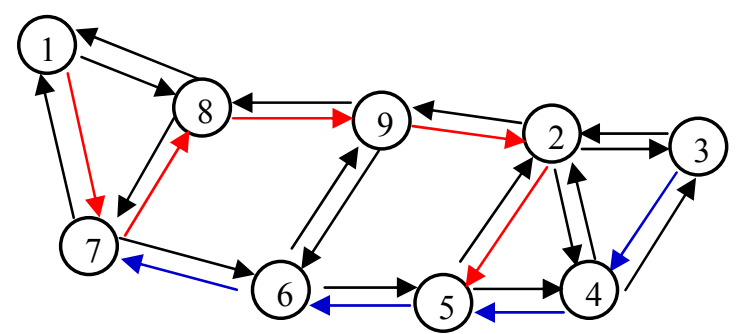

Figure 7. the Transmission of Period 7

Note. HA: $1 \rightarrow 7 \rightarrow 8 \rightarrow 9 \rightarrow 2 \rightarrow 5$ (218), $3 \rightarrow 4 \rightarrow 5 \rightarrow 6$ $\rightarrow 7$ (298); Opt: $1 \rightarrow 8 \rightarrow 9 \rightarrow 2 \rightarrow 5$ (171), $3 \rightarrow 4 \rightarrow 5 \rightarrow$ $6 \rightarrow 7(298)$

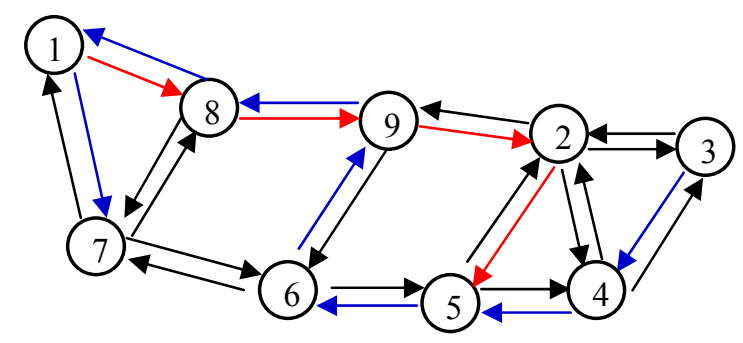

Figure 2. the Transmission of Period 2

Note. HA: $1 \rightarrow 8 \rightarrow 9 \rightarrow 2 \rightarrow 5$ (359), $3 \rightarrow 4 \rightarrow 5 \rightarrow 6 \rightarrow 9$ $\rightarrow 8 \rightarrow 1 \rightarrow 7$ (204); Opt: $1 \rightarrow 8 \rightarrow 9 \rightarrow 2 \rightarrow 5$ (359), $1 \rightarrow$ $7 \rightarrow 6 \rightarrow 5(270), 3 \rightarrow 4 \rightarrow 5 \rightarrow 6 \rightarrow 7(194)$

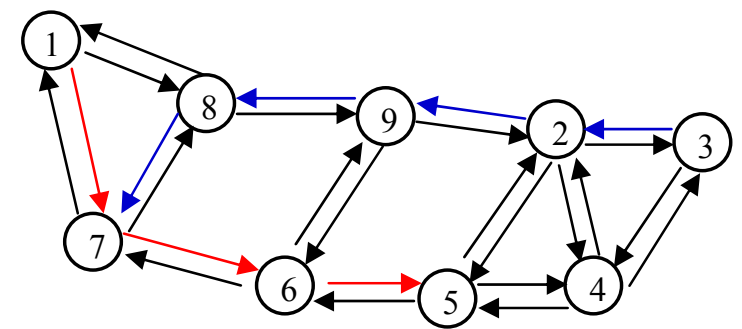

Figure 4. the Transmission of Period 4

Note. HA: $1 \rightarrow 7 \rightarrow 6 \rightarrow 5$ (349), $3 \rightarrow 2 \rightarrow 9 \rightarrow 8 \rightarrow 7$ (161); Opt: $1 \rightarrow 8 \rightarrow 9 \rightarrow 2 \rightarrow 5$ (155), $3 \rightarrow 4 \rightarrow 5 \rightarrow 6 \rightarrow$ $7(108)$

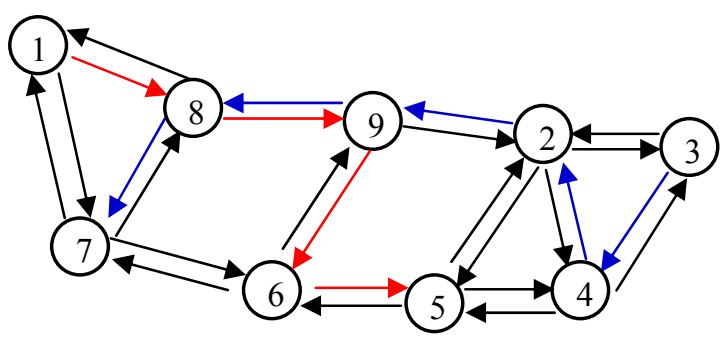

Figure 6. the Transmission of Period 6

Note. HA: $1 \rightarrow 8 \rightarrow 9 \rightarrow 6 \rightarrow 5$ (283), $3 \rightarrow 4 \rightarrow 2 \rightarrow 9 \rightarrow 8$ $\rightarrow 7$ (154);Opt: $1 \rightarrow 8 \rightarrow 9 \rightarrow 2 \rightarrow 5$ (248), $3 \rightarrow 4 \rightarrow 5 \rightarrow$ $6 \rightarrow 7(136)$

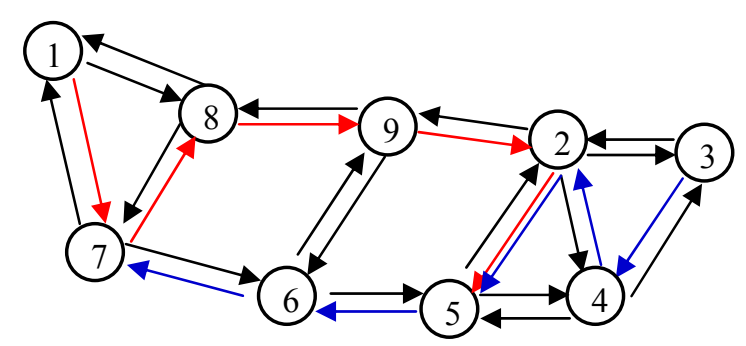

Figure 8. the Transmission of Period 8

Note. HA: $1 \rightarrow 7 \rightarrow 8 \rightarrow 9 \rightarrow 2 \rightarrow 5$ (129), $3 \rightarrow 4 \rightarrow 2 \rightarrow 5$ $\rightarrow 6 \rightarrow 7$ (241); Opt: $1 \rightarrow 8 \rightarrow 9 \rightarrow 2 \rightarrow 5(160) 3 \rightarrow 4 \rightarrow 5$ $\rightarrow 6 \rightarrow 7(220)$ 
Table 3. The network traffic of every time period

\begin{tabular}{cccccccccc}
\hline & T1 & T2 & T3 & T4 & T5 & T6 & T7 & T8 & total \\
\hline HA(1-5) & 232 & 359 & 365 & 349 & 265 & 283 & 218 & 129 & 2200 \\
Opt(1-5) & 199 & 629 & 488 & 155 & 150 & 248 & 171 & 160 & 2200 \\
HA(3-7) & 449 & 204 & 159 & 161 & 334 & 154 & 298 & 241 & 2000 \\
Opt(3-7) & 539 & 194 & 231 & 108 & 274 & 136 & 298 & 220 & 2000 \\
\hline
\end{tabular}

Note. $\operatorname{cost}(\mathrm{HA})=20071.186, \operatorname{cost}(\mathrm{Opt})=17260.5,|\operatorname{cost}(\mathrm{HA})-\operatorname{cost}(\mathrm{Opt})| / \operatorname{cost}(\mathrm{Opt})=16.28 \%$.

\subsection{Results Analysis}

According to the Figure 1 8 and table 3, the routing of the optimal solution is almost multi-path transmission while the heuristic algorithm is single path. Although the cost of the heuristic is 16.28 percent higher than the optimal algorithm, the heuristic algorithm is superior in computing speed and the single path resulting from heuristic algorithm occupies fewer links in the network topology for the non-real-time network traffic. From the perspective of the time complexity, global optimization gotten by interior point (Monteiro \& Adler, 1989) requires $O\left((k|E| \cdot|T|)^{3.5} L\right)$ time where $L$ is a number relative to the scale of the problem while the running time of heuristic algorithm is in $O\left(n^{2} T\right)$, noting that $n$ is the request number. However, when the differences of link cost are little, the effect of heuristic algorithm is more excellent than the optimal algorithm.

\section{Conclusions}

In this paper, we propose a heuristic algorithm to transmit the non-real-time network traffic in a particular network topology. When getting the information of the link bandwidth based on the network traffic prediction, residual bandwidth with the existed network topology constructs the residual network which serves the non-real-time network traffic transmission. The optimal solution on the basis of global optimization is achieved by solving the linear programming with interior point algorithm so that most of the routings are multi-path transmission. By contrast, the heuristic algorithm approximating greedy strategy gains the single path transmission which saves the link resources with low time complexity and not exceeding the optimal cost too much. In SDN, the management and maintenance of the network become more flexible and easy for operation.

\section{Acknowledgments}

This work is supported by the National Natural Science Foundation of China (71171189), the key project of the National Natural Science Foundation (11331012), 973 project (2011CB706901) and Huawei Technology Co. Ltd.

\section{References}

Bing, Q., Lu, J., \& Long, Y. (2008, October). An Improved Ant Algorithm for Network Traffic Control. In Wireless Communications, Networking and Mobile Computing, 2008. WiCOM'08. 4th International Conference on (pp. 1-4). IEEE. http://dx.doi.org/10.1109/WiCom.2008.1053

Box, G. E., Jenkins, G. M., \& Reinsel, G. C. (2013). Time series analysis: forecasting and control. John Wiley \& Sons.

Cristianini, N., \& Shawe, T. J. (2000). An introduction to support vector machines and other kernel-based learning methods. Cambridge university press.

Dassouki, K., Debar, H., Safa, H., \& Hijazi, A. (2013, June). A TCP delay-based mechanism for detecting congestion in the Internet. In Communications and Information Technology (ICCIT), 2013 Third $\begin{array}{lllll}\text { International } \quad \text { Conference } & \text { on } & \text { 141-145). }\end{array}$ http://dx.doi.org/10.1109/ICCITechnology.2013.6579538

Floyd, S., \& Gummadi, R. (2001). Adaptive RED: An algorithm for increasing the robustness of REDs Active Queue Management. Retrieved from http://www.icir.org/floyd/papers.html

Floyd, S., \& Jacobson, V. (1993). Random early detection gateways for congestion avoidance. Networking, IEEE/ACM Transactions on, 1(4), 397-413. http://dx.doi.org/10.1109/90.251892

Hao, K., Jin, Z., \& Hao, P. (2012, November). Study on Internet Congestion Control Algorithm. In Intelligent Ne tworks and Intelligent Systems (ICINIS), 2012 Fifth International Conference on (pp. 138-141). IEEE. http: //dx.doi.org/10.1109/ICINIS.2012.21

Huerta, M., Hesselbach, X., \& Fabregat, R. (2006, August). An Approach to Optimizations Links Utilization in 
MPLS Networks. In Computing in the Global Information Technology, 2006. ICCGI'06. International Multi-Conference on (pp. 58-58). IEEE. http://dx.doi.org/10.1109/ICCGI.2006.9

Jia, W. K., \& Wang, L. C. (2013). A Unified Unicast and Multicast Routing and Forwarding Algorithm for Software-Defined Datacenter Networks. Selected Areas in Communications, IEEE Journal on, 31(12), 2646-2657. http://dx.doi.org/10.1109/JSAC.2013.131206

Kantz, H., \& Schreiber, T. (2004). Nonlinear time series analysis (Vol. 7). Cambridge university press.

Leland, W. E., Taqqu, M. S., Willinger, W., \& Wilson, D. V. (1993, October). On the self-similar nature of Ethernet traffic. In ACM SIGCOMM Computer Communication Review (Vol. 23, No. 4, pp. 183-193). ACM. http://dx.doi.org/10.1145/167954.166255

Lin, D., \& Morris, R. (1997, October). Dynamics of random early detection. In ACM SIGCOMM Computer Communication Review (Vol. 27, No. 4, pp. 127-137). ACM. http://dx.doi.org/10.1145/263109.263154

McKeown, N., Anderson, T., Balakrishnan, H., Parulkar, G., Peterson, L., Rexford, J., ... \& Turner, J. (2008). OpenFlow: Enabling innovation in campus networks. ACM SIGCOMM Computer Communication Review, 38(2), 69-74. http://dx.doi.org/10.1145/1355734.1355746

Moghaddam, M. H. Y. (2010, August). A fuzzy Active Queue Management mechanism for Internet congestion control. In Advanced Computational Intelligence (IWACI), 2010 Third International Workshop on (pp. 203-208). IEEE. http://dx.doi.org/10.1109/IWACI.2010.5585186

Monteiro, R. D., \& Adler, I. (1989). Interior path following primal-dual algorithms. Part I: Linear programming. Mathematical programming, 44(1-3), 27-41. http://dx.doi.org/10.1007/BF01587075

Open Networking Foundation. (2012). Software-defined networking: The new norm for networks. Open Networking Foundation (ONF) White Paper. Retrieved from https://www.opennetworking.org/sdn-resources/sdn-library/whitepapers

Ott, Teunis J., Lakshman, T. V., \& Wong, L. H. (1999). "Sred: stabilized red." INFOCOM'99. Eighteenth Annual Joint Conference of the IEEE Computer and Communications Societies. Proceedings. IEEE. Vol. 3. IEEE, 1999. http://dx.doi.org/10.1109/INFCOM.1999.752153

Papadimitriou, D., Welzl, M., Scharf, M., \& Briscoe, B. (2011). Open research issues in Internet congestion control. Internet Research Task Force (IRTF), RFC, 6077. Retrieved from $\mathrm{http}: / /$ www.hjp.at/doc/rfc/rfc6077.html

Wijnants, M., \& Lamotte, W. (2008, January). Managing client bandwidth in the presence of both real-time and non real-time network traffic. In Communication Systems Software and Middleware and Workshops, 2008. COMSWARE 2008. 3rd International Conference on (pp. 442-450). IEEE. http://dx.doi.org/10.1109/COMSWA.2008.4554454

\section{Copyrights}

Copyright for this article is retained by the author(s), with first publication rights granted to the journal.

This is an open-access article distributed under the terms and conditions of the Creative Commons Attribution license (http://creativecommons.org/licenses/by/3.0/). 